\title{
2. No Mutation of the Catalase Gene in Cancer Patients
}

\author{
By Yosuke Kishimoto, Yoshinori Murakami, Kenshi HaYashi, \\ Shigeo Takahara, M. J. A., ${ }^{*}$ Takashi SugimurA, M. J. A., \\ and Takao SEKIYA \\ Oncogene Division, National Cancer Center Research \\ Institute, Tokyo 104 \\ (Communicated by Takashi Sugrmura, M. J. A., Feb. 12, 1991)
}

\begin{abstract}
The involvement of active oxygens in the genesis of human cancers has been proposed, and reduced catalase activity in cancer cells has been reported. To elucidate the possible involvement of aberrations of the catalase gene in carcinogenesis, we examined DNAs from tissues of cancer patients by single-strand conformation polymorphism analysis of polymerase chain reaction products. No mutation of the catalase gene was observed in 20 lung cancers analyzed or in noncancerous portions of the liver of 11 patients with hepatocellular carcinoma. These findings suggest that the catalase gene is not involved significantly, if at all, in tumorigenesis.
\end{abstract}

Key words: Catalase gene; lung cancer; hepatocellular carcinoma; single-strand conformation polymorphism analysis.

Catalase is an enzyme catalyzing the reaction in which hydrogen peroxide is converted to water and oxygen. This enzyme is thought to have biological roles in defense reactions against potentially harmful effects of active oxygens generated by a wide variety of biological processes in cells. ${ }^{1)}$ Recently, the possible involvement of active oxygens in human carcinogenesis has been proposed, ${ }^{2)}$ and differences in catalase activity in normal and cancerous cells have been reported. ${ }^{3)-5)}$ In particular, reduced catalase activity has been observed in both cancerous and noncancerous portions of the liver of patients with hepatocellular carcinoma. ${ }^{5}$ ) Thus it is possible that mutations of the catalase gene that reduce the enzyme activity may result in predisposition for cancer.

Recently, a point-mutation involved in acatalasemia in a Japanese patient has been identified. ${ }^{6}$ ) We also identified the same point-mutation of the catalase gene in other Japanese patients with acatalasemia and in one of the two catalase alleles in a patient with hypocatalasemia who has an intermediate level of enzyme activity (our unpublished results). To elucidate more frequent point-mutations of the catalase gene in cancer patients than in normal individuals, we investigated DNAs from lung cancer tissues and noncancerous portions of the liver of patients with hepatocellular carcinoma by single-strand conformation polymorphism analysis of polymerase chain reaction products (PCR-SSCP analysis). ${ }^{7), 8)}$

Materials and methods. DNAs were isolated from cancerous portions of the lung from 20 patients with lung cancer for analysis of possible somatic and also germ line mutations and noncancerous portions of the liver from 11 patients with hepatocellular carcinoma for analysis of germ line mutations by the method of Blin and Stafford. ${ }^{9)}$ DNA was also isolated from peripheral blood lymphocytes of an acatalasemic patient, who was the same individual examined previously by Wen et $a l .{ }^{6)}$ Of the 20 lung cancers studied, 10 were

*) Present address: 2-27 Hirose-cho, Okayama City 700, Japan. 
adenocarcinomas, 4 were squamous cell carcinomas, 3 were large cell carcinomas, 2 were small cell carcinomas and one was an adenoid cystic carcinoma. The DNAs isolated were subjected to PCR-SSCP analysis for detection of genetic changes of the catalase gene as described previously. ${ }^{8}$ The regions amplified using synthetic DNA primers of 20 oligonucleotides are indicated in Fig. 1. The nucleotide sequences of DNA fragments were determined by the asymmetric PCR method. ${ }^{10)}$

Results. Wen et al. identified a $\mathrm{G}$ to $\mathrm{A}$ transversion at the 5 th position of the intron between exons 4 and 5 (intron 4) as the causal point-mutation of the catalase gene in a Japanese acatalasemic patient and also reported polymorphic nucleotide substitutions at 6 positions of the gene in normal individuals (Fig. 1). ${ }^{6}$ ) We amplified 13 regions of the catalase gene by the PCR. The DNA fragments thus obtained, containing coding exons 1 to 13 (Fig. 1), were subjected to SSCP analysis. ${ }^{7), 8}$ By PCR-SSCP analysis, causal point-mutations and all polymorphic nucleotide substitutions could be detected as mobility shifts of DNA fragments on polyacrylamide gel electrophoresis. However, two polymorphic changes in introns 1 and 7 were not amplified in the system used in this study (Fig. 1). Nucleotide substitutions giving SSCPs were confirmed by nucleotide sequence determination of corresponding fragments.

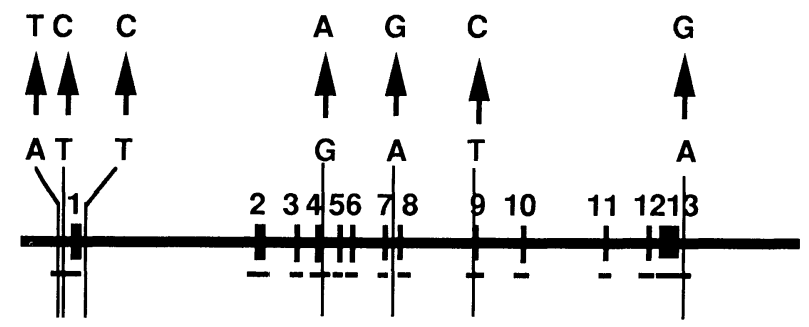

Fig. 1. Structure of the human catalase gene and the regions analyzed. Closed boxes with numbers are coding exons of the gene. Vertical lines indicate positions where polymorphic nucleotide substitutions shown by arrows are observed. ${ }^{6)}$ The nucleotide substitution indicated in the intron between exon 4 and 5 is the causal mutation reported by Wen $e t a l{ }^{6)}$

Using this PCR-SSCP method, we analyzed the DNAs from 31 surgical specimens from cancer patients, 20 specimens of tumor tissues of lung cancer patients and 11 specimens of noncancerous portions of the liver from patients with hepatocellular carcinoma. Fragment 4, carrying exon 4 and the position of the causal point-mutation for acatalasemia in intron 4, was amplified from DNAs of cancerous tissue of a lung cancer, a noncancerous portion of the liver of a hepatocellular carcinoma patient, and peripheral blood lymphocytes of an acatalasemic patient. The mobilities of single-stranded DNAs of these fragments are compared in Fig. 2. The results clearly indicate that the mobility of fragment 4 from acatalasemic DNA carrying the point-mutation differed from those of the DNAs of cancer patients. The results of analyses of the same fragment 4 from 20 lung cancer DNAs and 11 DNA samples from noncancerous portions of the liver of hepatocellular carcinoma patients are shown in Fig. 3. None of the 31 DNAs had any base substitution in the nucleotide sequence of this fragment (Fig. 3). Twelve other fragments were similarly analyzed. Although polymorphic nucleotide substitutions at 21 nucleotides upstream of the transcription initiation site (A to T), at 20 nucleotides upstream of the initiation codon ( $\mathrm{T}$ to $\mathrm{C}$ ), at codon 388 in exon 9 ( $\mathrm{T}$ to $\mathrm{C}$ ) and at 50 nucleotides downstream from the polyadenylation signal (A to G) (Fig. 1) were detected in 23, 26, 30 and 21, respectively, of 62 alleles (31 specimens), no aberration of the fragments was observed in 


\section{3}

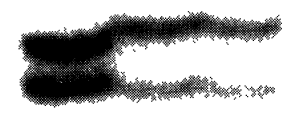

Fig. 2. PCR-SSCP analysis of fragment 4 carrying exon 4 and flanking regions of introns. Genomic DNAs from leukocytes of an acatalasemic patient (lane 1), surgical specimens of lung cancer (lane 2) and a noncancerous portion of the liver from a patient with hepatocellular carcinoma (lane 3) were subjected to PCR-SSCP analysis using a pair of primers flanking exon 4 of the catalase gene.

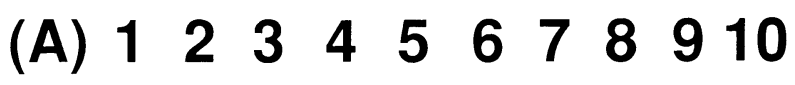

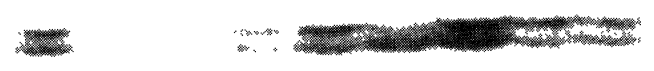

\section{0}

\section{(B) 1234567891011}

Fig. 3. PCR-SSCP analysis of fragment 4 from surgical specimens of human organs with cancers. DNAs from 20 surgical specimens of lung cancer (A) and noncancerous portions of the liver from 11 hepatocellular carcinoma patients (B) were analyzed. The samples analyzed in lane 2 and 3 in Fig. 2 were included in lane 1 (A) and lane 1 (B), respectively. 
any DNA specimen (data not shown). From these results, we conclude that the catalase gene is not involved significantly, if at all, in mediating carcinogenesis or predisposition to carcinogenesis in human cells.

Discussion. In eukaryotic cells, peroxisomes contain catalase together with oxidases. Many studies have revealed a reduced number of peroxisomes in tumors, and poorly differentiated or undifferentiated hepatocellular carcinomas are reported to contain no peroxisomes. ${ }^{11), 12)}$ Furthermore, the peroxisomes of rapidly growing, undifferentiated hepatoma are reported to contain no catalase activity. ${ }^{3)}$ Patients with malignant lymphomas and acute myeloid leukemias were found to have significantly decreased catalase activity. ${ }^{4}$ ) The catalase activity in both cancerous and noncancerous portions of the liver of patients with hepatocellular carcinoma was also reported to be significantly decreased. ${ }^{5)}$ From these observations, impairement of the system for scavenging active oxygens has been suggested to result in predisposition to carcinogenesis in humans. These findings suggest that there may be a mutation of the catalase gene that reduces the enzyme activity in human cancers or tissues of cancer patients. In this connection, it is noteworthly that Vuillaume et al. reported that increase of activated iorms of oxygen, mainly hydroxyradicals, due to decrease of anti-oxidant substances by deficiency of the gene for the anti-oxidative enzyme led to DNA damage causing carcinogenesis. ${ }^{13)}$ However, the relationship between genetic changes of the catalase gene and the occurrence of cancer have not been studied sufficiently. In this work, PCR-SSCP analysis of DNAs from 31 surgical specimens showed no mutation of the catalase gene specific to tumors, or any mutation of the gene in normal cells of cancer patients causing predisposition for cancers.

Acknowledgments. This work was supported in parts by a Grant-in-Aid for a Comprehensive 10-Year Strategy for Cancer Control from the Ministry of Health and Welfare, and grants from the Ministry of Education, Science and Culture, the Special Coordination Fund of the Science and Technology Agency of Japan, and a Grant-in-Aid from the Japan Academy. Y. Kishimoto is a recipient of a Research Resident Fellowship from the Foundation for Promotion of Cancer Research.

\section{References}

1) Oberley, L. W., and Buettner, G. R. (1979): Cancer Res., 39, 1141-1149.

2) Weitzman, S. A. et al. (1985): Science, 227, 1231-1233.

3) Mochizuki, Y. et al. (1971): Cancer Res., 31, 763-773.

4) Saito, T. et al. (1984): Cancer Lett., 24, 141-146.

5) Bellisola, G. et al. (1987): Clin. Biochem., 20, 415-417.

6) Wen, J. K. et al. (1990): J. Mol. Biol., 211, 383-393.

7) Orita, M. et al. (1989): Proc. Natl. Acad. Sci. USA, 86, 2766-2770.

8) - (1989): Genomics, 5, 874-879.

9) Blin, N., and Stafford, D. M. (1976): Nucl. Acids Res., 3, 2303-2308.

10) Gyllensten, U. B., and Erlich, H. A. (1988): Proc. Natl. Acad. Sci. USA, 85, 7652-7656.

11) Ma, M. H., and Blackburn, C. R. B. (1973): Cancer Res., 33, 1766-1774.

12) Mochizuki, Y. (1983): Metabolism and Disease, 20, 1105-1114.

13) Vuillaume, M. (1987): Mutat. Res., 186, 43-72. 